\title{
3D modified wavelet block tree coding for hyperspectral images
}

\author{
Shrish Bajpai ${ }^{1}$, Harsh Vikram Singh ${ }^{2}$, Naimur Rahman Kidwai ${ }^{3}$ \\ ${ }^{1}$ Electronic Engineering Department, A.K.T.U., India \\ ${ }^{2}$ Electronic Engineering Department, K.N.I.T., India \\ Department of ECE, Integral University, India
}

\begin{tabular}{|c|c|}
\hline Article Info & ABSTRACT \\
\hline Article history: & A novel wavelet-based efficient hyperspectral image compression scheme for \\
\hline Received Jan 4, 2019 & low memory sensors has been proposed. The proposed scheme uses the 3D \\
\hline Revised Feb 23, 2019 & $\begin{array}{l}\text { dyadic wavelet transform to exploit intersubband and intrasubband } \\
\text { correlation among the wavelet coefficients. By doing the reconstruction of }\end{array}$ \\
\hline Accepted Mar 10, 2019 & the transform image cube, taking the difference between the frames, it \\
\hline Keywords: & $\begin{array}{l}\text { complexity of the hyperspectral compression schemes in comparison with } \\
\text { other state-of-the-art compression schemes. }\end{array}$ \\
\hline
\end{tabular}

Compression

Hyperspectral image

Set partition compression

Transform coding

Wavelet transform

Copyright $(2019$ Institute of Advanced Engineering and Science. All rights reserved.

\section{Corresponding Author:}

Shrish Bajpai,

Electronics Engineering Department,

A.K.T.U. Campus,

Sector-11, Jankipuram Extension, Lucknow, India - 226031.

Email: shrishbajpai@gmail.com

\section{INTRODUCTION}

Hyperspectral Imaging (HSI) sensors capture the hundreds of continuous image frames for a single scene in the spectral range of 400 nanometers to 2500 nanometers with a nominal spectral sepration between the two frames of 10 nanometers. A single pixel of the hyperspectral image is represented by the multiple values which is correspondence to the associated wavelength,-contains the useful spectral information about the pixel [1].

This spectral information acquired in hundreds of bands allows recognizing any small change in the scene. Due to above-mentioned properties of HSI, it is used in the multiple applications such as agriculture, biotechnology, environmental monitoring, food product analysis, forensic science, material identification, medical diagnose, mineral mapping \& extraction, pharmaceuticals, surface investigation, remote sensing, security and defence services, space science, township planning, verification of documents etc [2].

Earth observation remote sensing is the one of the most remarkable application that takes the benefit of the information obtained from HSI sensors to identify, measure, and monitor constituents of the Earth's interior, exterior and it's climate. Hyperspectral remote sensing image can be viewed as a 3D data cube, with first two dimensions representing spatial coordinates on the Earth's surface, while the third dimension representing the frequency. Due to the large size of the hyperspectral image \& limited onboard memory availability of HSI sensor, it is necessary to apply the image compression scheme to save onboard hardware memory capacity, reduce network downlink transmission bandwidth and time [3-4]. 
The hyperspectral image compression scheme is the process of encoding information using fewer bits or other information-bearing units than an unencoded representation. Performance parameters \& compression ratio of hyperspectral images compression is stated in terms of bit per pixel per band (bppbp).

There are many compression schemes are proposed for hyperspectral images which can be classified into three categories: predictive coding, vector quantization, and transform-based compression schemes. Predictive coding schemes are based on the elimination of the inter-pixel redundancies of the closely spaced pixel by extracting and coding the new information in each pixel. This new information is the difference between the actual and predicted value of that pixel. It can be two type lossy or lossless. In the image compression scenario, the prediction of a hyperspectral image pixel for a current frame or group of pixels of current frame maybe drive from the previously transmitted pixels of that frame. The predicted pixel value is subtracted from the original pixel and a different signal is obtained. Differential Pulse Code Modulation (DPCM) is commonly used in predictive coding for image compression. Vector Quantization (VQ) is a lossy image compression scheme. VQ is a process of mapping multiple vectors into a single vector that has a smaller value. A vector of the image represents the small sub-image or a block of pixels. In VQ, it treats one entire sub-image as a single entity and quantized it by reducing total number of bits required to represent the sub-image. It is done by using a code book which stores the fix sets of vector and then coding the sub-image is performed by using the codebook.

Transform based compression scheme consist the two stages: transform and coding stage. The transform stage compact the energy of the hyperspectral image into the few coefficients of spatial and spectral domain. The coding stage of the transformed image is coded according to the values of the transform image coefficients [5]. The wavelet transform is widely employed due to its excellent clustering in time and space, computationally fast, it compresses images without appreciable degradation. 3D wavelet transform is applied to the 3D HSI cube using 1D wavelet transform employed in all direction in the row, column \& width fashion. Obtained wavelet coefficients are arranged in the pyramidal shape in which high energy coefficients are placed at the top. 3D dyadic wavelet transform is a mathematical way of encoding information in the layered structure according to the level of detail [6]. A single level 3D-Wavelet Transform divides the hyperspectral image in eight non-overlapping coefficient band known as LLL, LLH, LHL, LHH, HLL, HLH, HHL \& HHH. Wavelet transform based hyperspectral image composition schemes are broadly divided into two groups: Zerotree hyperspectral image compression schemes and Zeroblock hyperspectral image compression schemes. Spatial orientation trees (SOTs) are formed by the group of wavelet coefficients corresponding to the same spatial location for the zerotree hyperspectral image compression schemes. A SOT is zerotree when there is no significant wavelet coefficient above than current threshold level. In zeroblock schemes, contiguous blocks are formed from the transform hyperspectral image by dividing it according to the level of the transform. The significance test is done for the individual block. A block is zeroblock when there is no significant coefficient with reference to the current threshold. The new category of the compression scheme is combining the features of both zerotree compression schemes and zero block compression schemes. Zeroblocks of the partitioned image are formed and then block trees are formed with the roots in the topmost subband in the zerotree fashion [7-8].

Present manuscript has four sections. Section II describes the detail version of the 3D-Modified Wavelet Block Tree Coding (3D-MWBTC) for hyperspectral image compression. Section III gives the comparative simulation results of proposed 3D-MWBTC with the 3D-SPECK [6], 3D-SPIHT [9], 3D-LSK [10] \& 3D-WBTC [11] for four available hyperspectral images [12] on the five different parameters peak signal-to-noise ratio (PSNR), new significant bit found in the first ten pass, cumulative number of bits generated in the first ten pass, memory consumed by compression schemes, encoding time \& decoding time.

\section{RESEARCH METHOD}

Each frame of the hyperspectral image corresponds to the same scene. There is a high level of correlation between the different nearly placed frames of the hyperspectral image [13, 14]. This correlation property is exploited and the reconstructed hyperspectral image is generated. The cube structure of 3D gives the superior performance than the cuboid structure because of the partition of the cube will give the similar 8 subcubes (same dimension). The proposed 3D-MWBTC generates an embedded bit-stream like 3DSPIHT $[9,15]$.

Let's consider a hyperspectral image cube of dimension ' $x$ ' is transformed with five levels 3D dynamic wavelet transform. The 3D dyadic wavelet transform exhibit the pyramid subband structure in which low frequency (high energy) terms are present at the top portion. This transform image cube (dimension ' $x$ ') goes for the reconstructed phase. In the reconstruction phase of the transform image cube, the cube is divided (spectral dimension) in the sets having the eight continuous frames. The first frame of the set is preserved without any change, but for the $2^{\text {nd }}, 3^{\text {rd }}$ till $8^{\text {th }}$ frame of the set, the changes are done with the 
difference of current frame and the previous frame. This is done for rest all frames present in the hyperspectral image cube. It has been observed that the performance characteristics have been obtained with taking the eight frames together is better than taking the 4 or 16 frames in a set together. After the reconstruction of the hyperspectral image cube the 3D-wavelet block tree coding (3D-WBTC) scheme is applied. This 3D- wavelet block tree scheme has the best features of tree base coding schemes and blockbased coding schemes which make it a perfect choice for the compression scheme. There are many benefits of the reconstruction of hyperspectral image cube it reduces the maximum value of the hyperspectral image cube, which let the elimination of the top bit plane. The elimination of the top bitplane increases the peak signal ratio value as well as it will also reduce the complexity of the composition scheme. 3D-MWBTC has many advantages over zerotree base compression schemes (eg. 3D-SPIHT). It is a block-based compression scheme which saves memory with reference to the pixel based compression schemes. Intersubband correlation and spectral band correlations are exploited due to the pyramid structure obtained after the 3D wavelet transform. It combines multiple zerotrees which may occur in highest priority bitplane which lead the creation of the zerotree with more coefficients.

3D-MWBTC has three order list name as, a list of insignificant block cubes (LIBC), list of insignificance block set cubes (LIBSC) and list of significant pixels (LSP). In the initialization phase, block cubes present in LLL band are added to the LIBC and those with the descendents are added to the LIBCS as type 'A' blocks. The LSP initialized with the empty list. Type 'A' blocks are those blocks who are not significant and their children are not significant to the current threshold. While type 'B' blocks are those blocks who are not significant but their children are significant.

The encoding process has two stages within each bitplane sorting followed by refinement phase. The encoding process starts from the most significant bit plane. In the sorting pass, first, it will check the LIBC list. If the block cube is present in the list, the significance of the block cube will be tested against the current threshold level. Only one bit is used for the significance of the block. If the block cube is found insignificant then, it is zero block and ' 0 ' is sent. The block cube will remain in the LIBC. There will be no further partitioning and no new bits will be generated for the block. If the block is found significant against the current threshold level, then it is non zero block and '1' is sent. A significant block is partitioned into the eight subcubes using octuple-tree partitioning rule. The partition operation recursively repeated until no further partition is needed or the smallest possible block cube size $(2 * 2 * 2)$. At this stage, eight coefficients and their significance are individually tested. If a coefficient is insignificant, then ' 0 ' is sent and the position is moved to LIBC as a single coefficient block. If the coefficient is significant than '1' is sent, a sign bit is also coded and this coefficient is moved to LSP. After performing the testing of all eight individual coefficients in a block cube, the current block cube is removed from the LIBC. Then encoder tests the block cube sets present in the LIBCS and performs the significance test for each block cube sets. Significant block cube sets are divided into the subsets while insignificant block cube sets remain in LIBCS.

A significant type ' $A$ ' set is partitioned into eight type ' $B$ ' sets and eight offspring block cubes. The type ' $B$ ' set is added to the end of the LIBCS while eight offspring block cubes are tested for their significance in the same manner as they are present in the LIBC. A significant type ' $\mathrm{B}$ ' block cube is partitioned into eight type ' $A$ ' block cube and all of them are added to the end of the LIBCS. After each sorting pass, all the coefficients present in the LSP before the starting of the current biplane, are refined with one bit. This process repeats the above process by decreasing the threshold level by the factor of 2 until the bit budget is available.

The decoder follows the same procedure as the encoder with no changes and an additional step of significance testing of coefficient or block cube sets to identify coefficient or block sets containing coefficients requiring refinement.

\section{RESULTS AND ANALYSIS}

The performance of the proposed compression scheme 3D-MWBTC is compared with the 3DSPECK [6], 3D-SPIHT [9], 3D-LSK [10] \& 3D-WBTC [11]. Parameters are evaluated on four different standard hyperspectral images which are Washington DC (1280x307x191), Culprit (250x190x224), Jasper Ridge $(100 \times 100 \times 224) \&$ Urban $(307 * 307 * 210)$. Hyperspectral images are taken in the size of the cube of the dimension 128. The padding has been performed with ' 0 ' for the hyperspectral images who does not have the cube shape of the mention dimension. Hyperspectral images are cropped from the initial to get the desired cube size. The simulation of the different hyperspectral images compression schemes have been implemented using the Matlab 2016A version and executed on Windows 8.1 operating system. Compression is needed to save the power of onboard sensors \& data transmission bandwith [16-17]. 


\subsection{Coding Efficency}

The coding efficiency of the hyperspectral images is measured by the parameter peak signal to noise ratio (PSNR). This parameter shows the quality of reconstructed hyperspectral images with reference to the original hyperspectral images. It is defined by equation (1) \& (2) [18].

$$
\text { PSNR }=10 \log _{10}\left[\frac{(\text { Maximum Signal Power })}{\text { MSE }}\right]
$$

where MSE is the mean square error of the reconstructed hyperspectral images with the original hyperspectral images. It is calculated with the equation (2).

$$
\operatorname{MSE}=\frac{1}{\mathrm{~N}_{\mathrm{pix}}} \sum_{\mathrm{x}, \mathrm{y}, \mathrm{z}}[\mathrm{f}(\mathrm{x}, \mathrm{y}, \mathrm{z})-\mathrm{g}(\mathrm{x}, \mathrm{y}, \mathrm{z})]^{2}
$$

Table 1 is the comparative performance of the proposed composition scheme with the other state of art hyperspectral image compression which is taken at the bppbp of 0.1 to 1 . It is clearly evident that the proposed compression scheme 3D-MWBTC is outperforms the compression schemes. This is due to the elimination of the highest bit plane of the hyperspectral images which leads the significant increment of the PSNR for all hyperspectral images. It has been observed that the proposed compression scheme 3D-MWBTC has been outperformed with other compression scheme but according to the maximum pixel value of the hyperspectral image cube. 3D-MWBTC is significantly better than the other compression scheme at low bit rates (bppbp). The main reason behind this performance is that when high priority bit planes are coded, the majority of coefficients are insignificant and proposed compression scheme combined a large volume of insignificant coefficient together represented by a single bit.

Table 1. PSNR (in db) comparison of M-3D-WBTC with 3D-SPECK [6], 3D-SPIHT [9], 3D-LSK [10], 3D-WBTC [11] at various bit rates for the four different hyperspectral images Washington DC,

\begin{tabular}{|c|c|c|c|c|c|c|c|c|c|c|}
\hline bpppb & 3D-SPECK & $\begin{array}{c}\text { 3D- } \\
\text { SPIHT }\end{array}$ & 3D-LSK & $\begin{array}{c}\text { 3D- } \\
\text { WBTC }\end{array}$ & $\begin{array}{l}\text { M-3D- } \\
\text { WBTC }\end{array}$ & $\begin{array}{c}\text { 3D- } \\
\text { SPECK }\end{array}$ & $\begin{array}{c}\text { 3D- } \\
\text { SPIHT }\end{array}$ & 3D-LSK & $\begin{array}{c}\text { 3D- } \\
\text { WBTC }\end{array}$ & $\begin{array}{l}\text { M-3D- } \\
\text { WBTC }\end{array}$ \\
\hline & \multicolumn{5}{|c|}{ Washington DC } & \multicolumn{5}{|c|}{ Jasper Ridge } \\
\hline 0.1 & 38.53 & 38.28 & 38.35 & 38.7 & 45.47 & 35.08 & 35.11 & 35.29 & 35.67 & 48.65 \\
\hline 0.2 & 41.54 & 41.34 & 41.29 & 41.72 & 47.81 & 39.35 & 39.13 & 39.40 & 39.60 & 52.47 \\
\hline 0.3 & 43.51 & 43.3 & 43.55 & 43.69 & 49.20 & 41.72 & 41.89 & 41.95 & 42.40 & 54.96 \\
\hline 0.4 & 45.26 & 45.11 & 44.79 & 45.45 & 51.61 & 44.52 & 44.41 & 44.55 & 44.81 & 57.41 \\
\hline 0.5 & 46.81 & 46.6 & 46.36 & 47.01 & 54.58 & 45.91 & 46.33 & 46.26 & 46.78 & 59.12 \\
\hline 0.6 & 48.45 & 48.24 & 48.42 & 48.63 & 55.42 & 48.17 & 48.19 & 48.41 & 48.62 & 61.01 \\
\hline 0.7 & 49.76 & 49.53 & 49.43 & 49.74 & 56.33 & 49.94 & 50.06 & 50.05 & 50.53 & 62.70 \\
\hline 0.8 & 51.12 & 50.84 & 50.7 & 51.29 & 57.02 & 51.13 & 51.74 & 51.71 & 52.20 & 63.52 \\
\hline 0.9 & 52.24 & 52.06 & 52.24 & 52.42 & 58.31 & 52.97 & 53.09 & 53.30 & 53.51 & 64.98 \\
\hline \multirow[t]{2}{*}{1} & 53.52 & 53.32 & 53.49 & 53.71 & 59.93 & 54.77 & 54.72 & 54.86 & 55.13 & 66.04 \\
\hline & \multicolumn{5}{|c|}{ Cuprite } & \multicolumn{5}{|c|}{ Urban } \\
\hline 0.1 & 25.64 & 24.67 & 25.65 & 25.57 & 33.01 & 57.04 & 56.94 & 57.04 & 57.02 & 62.35 \\
\hline 0.2 & 30.92 & 29.44 & 30.88 & 31.03 & 39.76 & 58.95 & 58.80 & 58.76 & 58.95 & 63.18 \\
\hline 0.3 & 34.55 & 33.36 & 34.55 & 34.58 & 43.87 & 60.43 & 60.29 & 60.43 & 60.42 & 64.90 \\
\hline 0.4 & 38.05 & 37.04 & 38.05 & 38.15 & 46.54 & 61.77 & 61.67 & 61.54 & 61.76 & 65.88 \\
\hline 0.5 & 41.27 & 40.51 & 41.32 & 41.37 & 48.32 & 62.95 & 62.79 & 62.69 & 62.94 & 66.30 \\
\hline 0.6 & 43.46 & 42.58 & 43.47 & 43.57 & 50.19 & 64.16 & 64.00 & 64.05 & 64.16 & 67.41 \\
\hline 0.7 & 45.55 & 45.00 & 45.78 & 45.57 & 51.51 & 65.37 & 65.27 & 65.35 & 65.37 & 68.84 \\
\hline 0.8 & 47.12 & 46.43 & 47.07 & 47.26 & 52.85 & 66.33 & 66.21 & 66.09 & 66.34 & 69.19 \\
\hline 0.9 & 48.74 & 47.95 & 48.75 & 48.85 & 54.26 & 67.36 & 67.25 & 67.03 & 67.36 & 70.26 \\
\hline 1 & 49.83 & 49.24 & 49.86 & 49.98 & 55.48 & 68.40 & 68.23 & 68.11 & 68.40 & 71.07 \\
\hline
\end{tabular}
Jasper Ridge, Cuprite \& Urban [12]

Table 2 represents the newly significant bits found in the first ten passes of the four different hyperspectral image compression schemes. It has been observed that propose compression scheme sucessfully identify more significant bits than the other hyperspectral image compression schemes due to the reconstructed hyperspectral image cube. This has been done by the proposed compression scheme by keeping the high PSNR values with reference to the other compression scheme. 
Table 2. New significant bit found in the first ten pass of 3D-SPECK [6], 3D-SPIHT [9], 3D-LSK [10], 3D-WBTC [11] at various bit rates for the four different hyperspectral images Washington DC, Jasper Ridge, Cuprite \& Urban [12]

\begin{tabular}{|c|c|c|c|c|c|c|c|c|c|c|}
\hline Pass & 3D-SPECK & 3D-SPIHT & 3D-LSK & $\begin{array}{c}\text { 3D- } \\
\text { WBTC }\end{array}$ & $\begin{array}{l}\text { M-3D- } \\
\text { WBTC }\end{array}$ & $\begin{array}{c}\text { 3D- } \\
\text { SPECK }\end{array}$ & $\begin{array}{c}\text { 3D- } \\
\text { SPIHT }\end{array}$ & 3D-LSK & $\begin{array}{c}\text { 3D- } \\
\text { WBTC }\end{array}$ & $\begin{array}{l}\text { M-3D- } \\
\text { WBTC }\end{array}$ \\
\hline & \multicolumn{5}{|c|}{ Washington DC } & \multicolumn{5}{|c|}{ Jasper Ridge } \\
\hline 1 & 17 & 17 & 17 & 17 & 22 & 7 & 7 & 7 & 7 & 12 \\
\hline 2 & 50 & 50 & 50 & 50 & 59 & 30 & 30 & 30 & 30 & 44 \\
\hline 3 & 65 & 65 & 65 & 65 & 84 & 71 & 71 & 71 & 71 & 84 \\
\hline 4 & 178 & 178 & 178 & 178 & 198 & 217 & 217 & 217 & 217 & 311 \\
\hline 5 & 533 & 533 & 533 & 533 & 612 & 636 & 636 & 636 & 636 & 818 \\
\hline 6 & 1786 & 1786 & 1786 & 1786 & 1918 & 1671 & 1671 & 1671 & 1671 & 1801 \\
\hline 7 & 5558 & 5558 & 5558 & 5558 & 6018 & 5602 & 5602 & 5602 & 5602 & 5948 \\
\hline 8 & 18159 & 18159 & 18159 & 18159 & 19185 & 18286 & 18286 & 18286 & 18286 & 20022 \\
\hline 9 & 48342 & 48342 & 48342 & 48342 & 51235 & 37407 & 37407 & 37407 & 37407 & 39948 \\
\hline \multirow{2}{*}{10} & 108519 & 77842 & 78941 & 71878 & 75356 & 72574 & 72574 & 72574 & 72574 & 77121 \\
\hline & \multicolumn{5}{|c|}{ Cuprite } & \multicolumn{5}{|c|}{ Urban } \\
\hline 1 & 5 & 5 & 5 & 5 & 8 & 33 & 33 & 33 & 33 & 59 \\
\hline 2 & 50 & 50 & 50 & 50 & 51 & 58 & 58 & 58 & 58 & 201 \\
\hline 3 & 219 & 219 & 219 & 219 & 255 & 70 & 70 & 70 & 70 & 297 \\
\hline 4 & 537 & 537 & 537 & 537 & 702 & 156 & 156 & 156 & 156 & 548 \\
\hline 5 & 5099 & 5099 & 5099 & 5099 & 5323 & 745 & 745 & 745 & 745 & 2073 \\
\hline 6 & 10324 & 10324 & 10324 & 10324 & 11002 & 3002 & 3002 & 3002 & 3002 & 9285 \\
\hline 7 & 22424 & 22424 & 22424 & 22424 & 23874 & 11305 & 11305 & 11305 & 11305 & 37475 \\
\hline 8 & 46681 & 46681 & 46681 & 46681 & 49128 & 35333 & 35333 & 35333 & 35333 & 92623 \\
\hline 9 & 79986 & 79986 & 79986 & 79986 & 82079 & 108506 & 108506 & 108506 & 108506 & 169220 \\
\hline 10 & 120660 & 120660 & 120660 & 112854 & 119756 & 252166 & 252166 & 252166 & 252166 & 275708 \\
\hline
\end{tabular}

\subsection{Memory}

Table 3 represents the memory requirement of the 3D-MWBTC for different bit per pixel per band rate (bppbp) with 3D-SPECK [6], 3D-SPIHT [9], 3D-LSK [10], 3D-WBTC [11]. As shown in the Table 3, 3D-MWBTC has less memory requirement with other hyperspectral image compression schemes. It is clear that memory requirement of all five compression scheme in top priority bitplane is more significant than lower low priority bitplane because at the lowest threshold level more sets will be significant and 3DMWBTC will result in more entries in LIBC.

Table 3. Memory (kb) use by the 3D-SPECK [6], 3D-SPIHT [9], 3D-LSK [10], 3D-WBTC [11] at various bit rates for the four different hyperspectral images Washington DC, Jasper Ridge, Cuprite \& Urban [12]

\begin{tabular}{|c|c|c|c|c|c|c|c|c|c|c|}
\hline \multirow[b]{2}{*}{ bppbp } & \multicolumn{5}{|c|}{ Washington DC } & \multicolumn{5}{|c|}{ Jasper Ridge } \\
\hline & 3D-SPECK & 3D-SPIHT & $\begin{array}{l}\text { 3D- } \\
\text { LSK }\end{array}$ & 3D-WBTC & $\begin{array}{l}\text { M-3D- } \\
\text { WBTC }\end{array}$ & 3D-SPECK & 3D-SPIHT & $\begin{array}{l}\text { 3D- } \\
\text { LSK }\end{array}$ & 3D-WBTC & $\begin{array}{l}\text { M-3D- } \\
\text { WBTC }\end{array}$ \\
\hline 0.1 & 243.84 & 263.28 & 512 & 250.09 & 241.30 & 241.36 & 245.86 & 512 & 245.83 & 227.25 \\
\hline 0.2 & 416.29 & 437.97 & 512 & 416.00 & 402.17 & 439.95 & 445.74 & 512 & 443.73 & 411.69 \\
\hline 0.3 & 701.05 & 628.55 & 512 & 704.03 & 635.99 & 541.29 & 555.28 & 512 & 549.26 & 512.66 \\
\hline 0.4 & 733.80 & 723.55 & 512 & 732.97 & 805.79 & 729.56 & 759.53 & 512 & 741.59 & 662.86 \\
\hline 0.5 & 1048.80 & 1060.50 & 512 & 1049.00 & 1075.85 & 821.57 & 808.88 & 512 & 827.94 & 799.90 \\
\hline 0.6 & 1191.10 & 1222.60 & 512 & 1195.40 & 1075.85 & 1099.85 & 1123.21 & 512 & 1106.71 & 985.66 \\
\hline 0.7 & 1191.90 & 1222.60 & 512 & 1195.60 & 1262.85 & 1099.85 & 1123.21 & 512 & 1106.71 & 1012.95 \\
\hline 0.8 & 1407.70 & 1415.30 & 512 & 1404.40 & 1587.01 & 1178.85 & 1192.71 & 512 & 1189.12 & 1087.48 \\
\hline 0.9 & 1702.50 & 1725.50 & 512 & 1704.60 & 1603.14 & 1443.24 & 1467.97 & 512 & 1450.32 & 1221.44 \\
\hline 1 & 1802.50 & 1826.70 & 512 & 1724.60 & 1669.57 & 1443.24 & 1467.97 & 512 & 1450.32 & 1221.44 \\
\hline bppbp & 3D-SPECK & 3D-SPIHT & $\begin{array}{c}\text { Cuprite } \\
\text { 3D- } \\
\text { LSK }\end{array}$ & 3D-WBTC & $\begin{array}{l}\text { M-3D- } \\
\text { WBTC }\end{array}$ & 3D-SPECK & 3D-SPIHT & $\begin{array}{l}\text { Urban } \\
\text { 3D- } \\
\text { LSK }\end{array}$ & 3D-WBTC & $\begin{array}{l}\text { M-3D- } \\
\text { WBTC }\end{array}$ \\
\hline 0.1 & 277.73 & 277.60 & 512 & 282.79 & 185.33 & 293.23 & 299.44 & 512 & 294.27 & 251.79 \\
\hline 0.2 & 414.52 & 434.27 & 512 & 417.15 & 248.51 & 478.12 & 529.82 & 512 & 483.45 & 534.85 \\
\hline 0.3 & 544.29 & 514.74 & 512 & 546.27 & 333.95 & 841.20 & 863.95 & 512 & 842.73 & 679.98 \\
\hline 0.4 & 601.93 & 576.49 & 512 & 594.45 & 496.66 & 841.20 & 866.95 & 512 & 842.73 & 981.90 \\
\hline 0.5 & 671.18 & 701.89 & 512 & 674.51 & 751.95 & 1076.59 & 1110.29 & 512 & 1076.96 & 981.90 \\
\hline 0.6 & 854.01 & 783.74 & 512 & 857.57 & 834.04 & 1419.37 & 1444.85 & 512 & 1424.83 & 1200.32 \\
\hline 0.7 & 854.02 & 783.74 & 512 & 857.57 & 885.65 & 1563.42 & 1586.39 & 512 & 1564.13 & 1477.50 \\
\hline 0.8 & 1065.30 & 964.62 & 512 & 1057.30 & 1003.91 & 1563.42 & 1586.39 & 512 & 1564.13 & 1477.50 \\
\hline 0.9 & 1158.50 & 1182.40 & 512 & 1159.50 & 1173.22 & 1590.25 & 1586.39 & 512 & 1589.81 & 1481.34 \\
\hline 1 & 1158.50 & 1182.40 & 512 & 1159.60 & 1173.22 & 1889.49 & 1906.35 & 512 & 1808.87 & 1747.06 \\
\hline
\end{tabular}




\subsection{Complexity Analysis}

Table 4 and Table 5 are the encoding and decoding time of the 3D-MWBTC with the comparative analysis of 3D-SPECK [6], 3D-SPIHT [9], 3D-LSK [10], 3D-WBTC [11]. This time has been calculated for different bit per pixel per band (bppb) values. The time of wavelet transform and inverse wavelet transform is not included in the encoding and decoding time. It has been cleared that the proposed scheme is taking less encoding and decoding time with reference to other compression schemes due to the low value of the highest pixel value present in the image cube.

Table 4. Encoding Time (sec) of the 3D-SPECK [6], 3D-SPIHT [9], 3D-LSK [10], 3D-WBTC [11] at various bit rates for the four different hyperspectral images Washington DC, Jasper Ridge, Cuprite \& Urban [12]

\begin{tabular}{|c|c|c|c|c|c|c|c|c|c|c|}
\hline bppbp & 3D-SPECK & 3D-SPIHT & $\begin{array}{l}\text { 3D- } \\
\text { LSK }\end{array}$ & 3D-WBTC & $\begin{array}{l}\text { M-3D- } \\
\text { WBTC }\end{array}$ & 3D-SPECK & $\begin{array}{c}\text { 3D- } \\
\text { SPIHT }\end{array}$ & $\begin{array}{l}\text { 3D- } \\
\text { LSK }\end{array}$ & 3D-WBTC & $\begin{array}{l}\text { M-3D- } \\
\text { WBTC }\end{array}$ \\
\hline & \multicolumn{5}{|c|}{ Washington DC } & \multicolumn{5}{|c|}{ Jasper Ridge } \\
\hline 0.1 & 24.98 & 7.47 & 0.77 & 6.51 & 5.17 & 21.10 & 7.62 & 0.93 & 6.36 & 5.15 \\
\hline 0.2 & 57.94 & 25.76 & 1.08 & 24.81 & 16.31 & 54.19 & 20.56 & 1.16 & 17.71 & 13.62 \\
\hline 0.3 & 92.12 & 37.47 & 1.53 & 32.03 & 45.25 & 100.62 & 39.43 & 1.49 & 42.77 & 26.12 \\
\hline 0.4 & 269.74 & 117.89 & 1.99 & 195.46 & 70.26 & 150.94 & 47.77 & 2.21 & 70.67 & 71.05 \\
\hline 0.5 & 414.80 & 140.13 & 2.53 & 194.19 & 77.97 & 315.28 & 101.56 & 2.64 & 182.38 & 78.88 \\
\hline 0.6 & 576.03 & 166.40 & 2.91 & 247.89 & 311.97 & 355.95 & 115.33 & 3.03 & 227.51 & 185.58 \\
\hline 0.7 & 887.48 & 405.70 & 3.16 & 625.02 & 404.48 & 426.13 & 232.29 & 3.15 & 480.85 & 325.46 \\
\hline 0.8 & 1130.50 & 474.16 & 3.80 & 710.20 & 456.71 & 585.74 & 382.31 & 3.69 & 676.43 & 362.20 \\
\hline 0.9 & 1334.60 & 555.72 & 4.04 & 746.00 & 592.91 & 701.17 & 415.02 & 3.98 & 771.90 & 450.15 \\
\hline \multirow[t]{2}{*}{1} & 1497.50 & 574.96 & 4.38 & 804.00 & 704.88 & 757.32 & 425.38 & 5.05 & 942.81 & 659.52 \\
\hline & \multicolumn{5}{|c|}{ Cuprite } & \multicolumn{5}{|c|}{ Urban } \\
\hline 0.1 & 17.26 & 6.29 & 0.85 & 4.69 & 4.48 & 15.64 & 6.79 & 1.58 & 6.85 & 6.15 \\
\hline 0.2 & 55.82 & 26.01 & 1.21 & 16.61 & 9.76 & 49.54 & 19.74 & 2.97 & 19.09 & 20.54 \\
\hline 0.3 & 107.91 & 45.52 & 1.98 & 39.06 & 16.95 & 85.71 & 48.89 & 4.11 & 26.36 & 45.59 \\
\hline 0.4 & 182.28 & 75.55 & 2.09 & 68.19 & 25.45 & 312.36 & 202.14 & 5.64 & 191.77 & 56.80 \\
\hline 0.5 & 276.14 & 95.44 & 2.19 & 93.32 & 36.70 & 416.21 & 198.11 & 7.07 & 253.64 & 232.62 \\
\hline 0.6 & 298.42 & 161.67 & 3.44 & 155.69 & 85.63 & 886.92 & 206.67 & 8.84 & 300.34 & 277.05 \\
\hline 0.7 & 438.80 & 179.18 & 3.85 & 202.24 & 135.15 & 605.09 & 211.86 & 9.48 & 371.65 & 294.26 \\
\hline 0.8 & 558.74 & 198.45 & 4.21 & 358.46 & 155.02 & 1125.15 & 541.76 & 11.09 & 788.74 & 548.23 \\
\hline 0.9 & 656.09 & 282.84 & 4.42 & 370.97 & 271.57 & 1542.71 & 774.09 & 12.69 & 1067.05 & 801.75 \\
\hline 1 & 905.06 & 364.00 & 4.97 & 652.46 & 481.80 & 1702.79 & 780.18 & 14.37 & 1184.45 & 857.14 \\
\hline
\end{tabular}

Table 5. Decoding Time (sec) of the 3D-SPECK [6], 3D-SPIHT [9], 3D-LSK [10], 3D-WBTC [11] at various bit rates for the four different hyperspectral images Washington DC, Jasper Ridge,

Cuprite \& Urban [12]

\begin{tabular}{|c|c|c|c|c|c|c|c|c|c|c|}
\hline bppbp & 3D-SPECK & 3D-SPIHT & $\begin{array}{l}\text { 3D- } \\
\text { LSK }\end{array}$ & 3D-WBTC & $\begin{array}{l}\text { M-3D- } \\
\text { WBTC }\end{array}$ & 3D-SPECK & $\begin{array}{c}\text { 3D- } \\
\text { SPIHT }\end{array}$ & $\begin{array}{l}\text { 3D- } \\
\text { LSK }\end{array}$ & 3D-WBTC & $\begin{array}{l}\text { M-3D- } \\
\text { WBTC }\end{array}$ \\
\hline & \multicolumn{5}{|c|}{ Washington DC } & \multicolumn{5}{|c|}{ Jasper Ridge } \\
\hline 0.1 & 17.42 & 6.12 & 0.71 & 5.03 & 3.52 & 15.31 & 7.41 & 0.75 & 4.57 & 3.43 \\
\hline 0.2 & 48.77 & 24.84 & 1.12 & 22.45 & 14.39 & 36.99 & 17.63 & 1.12 & 15.32 & 11.35 \\
\hline 0.3 & 75.39 & 34.77 & 1.50 & 28.50 & 43.39 & 84.67 & 37.14 & 1.43 & 39.95 & 22.45 \\
\hline 0.4 & 264.22 & 106.26 & 1.72 & 180.43 & 69.66 & 128.79 & 44.96 & 2.08 & 68.97 & 70.31 \\
\hline 0.5 & 339.07 & 135.44 & 2.17 & 191.65 & 75.40 & 290.76 & 98.51 & 2.61 & 178.42 & 74.42 \\
\hline 0.6 & 532.38 & 130.56 & 2.64 & 244.64 & 306.66 & 330.90 & 155.55 & 2.80 & 229.76 & 181.47 \\
\hline 0.7 & 807.58 & 427.07 & 2.64 & 558.00 & 400.22 & 386.40 & 232.15 & 3.02 & 432.08 & 319.98 \\
\hline 0.8 & 1058.10 & 468.88 & 3.08 & 675.31 & 469.02 & 487.76 & 382.18 & 3.50 & 608.44 & 356.30 \\
\hline 0.9 & 1142.30 & 486.23 & 3.17 & 725.00 & 597.09 & 667.35 & 402.73 & 3.55 & 673.47 & 461.30 \\
\hline \multirow[t]{2}{*}{1} & 1289.66 & 503.96 & 3.68 & 874.00 & 689.27 & 726.86 & 421.31 & 4.28 & 923.14 & 642.32 \\
\hline & \multicolumn{5}{|c|}{ Cuprite } & \multicolumn{5}{|c|}{ Urban } \\
\hline 0.1 & 13.38 & 5.00 & 0.66 & 3.14 & 2.78 & 11.78 & 4.28 & 1.50 & 4.76 & 4.85 \\
\hline 0.2 & 46.66 & 22.08 & 0.99 & 14.57 & 9.76 & 41.59 & 16.83 & 2.39 & 16.38 & 18.32 \\
\hline 0.3 & 93.70 & 40.23 & 1.77 & 35.41 & 16.95 & 72.29 & 42.50 & 3.24 & 23.17 & 43.17 \\
\hline 0.4 & 162.48 & 70.08 & 1.87 & 65.81 & 25.45 & 292.17 & 218.31 & 4.65 & 194.48 & 53.97 \\
\hline 0.5 & 236.13 & 88.29 & 2.02 & 91.52 & 36.70 & 388.44 & 198.82 & 5.35 & 243.21 & 228.35 \\
\hline 0.6 & 319.20 & 160.90 & 2.86 & 148.97 & 85.63 & 487.20 & 197.21 & 6.14 & 294.68 & 271.86 \\
\hline 0.7 & 435.00 & 175.79 & 3.10 & 196.82 & 135.15 & 592.70 & 199.07 & 8.20 & 365.65 & 289.21 \\
\hline 0.8 & 525.89 & 195.33 & 3.79 & 315.96 & 155.02 & 1066.72 & 528.92 & 8.80 & 771.74 & 546.03 \\
\hline 0.9 & 599.22 & 273.50 & 4.04 & 366.90 & 271.57 & 1506.41 & 749.75 & 9.37 & 1052.34 & 798.03 \\
\hline 1 & 884.44 & 346.64 & 4.49 & 595.98 & 481.80 & 1661.79 & 3908.40 & 10.04 & 1173.39 & 853.29 \\
\hline
\end{tabular}




\section{CONCLUSION}

The work has been demonstrated that the high signal to noise ratio can be achieved by the proposed hyperspectral image compression scheme with limited loss of critical information according to the compression point of view. In this scheme the hyperspectral image cube is reconstructed and the top priority bit plane is eliminated by the taking difference between the consecutive frames of hyperspectral image cube. This compression scheme is suited for the sensors which needs high PSNR at the low bit per pixel per band rate. It has the relatively less coding memory requirement with the other compression scheme as shown in the Table 3.

\section{REFERENCES}

[1] B. K. Mohan and A. Porwal, "Hyperspectral Image Processing and Analysis," Current Science, vol. 108, pp. 833841, Mar 2015.

[2] S. Bajpai, et al., "Feature extraction \& classification of hyperspectral images using singular spectrum analysis \& multinomial logistic regression classifiers," International Conference on Multimedia, Signal Processing and Communication Technologies (IMPACT), pp. 97-100, Nov 2017.

[3] G. Motta, et al., "Hyperspectral data compression," Springer Science \& Business Media, 2006.

[4] J. Uthayakumar, et al., "A Survey on Data Compression Techniques: From the Perspective of Data Quality, Coding Schemes, Data Type and Applications," Journal of King Saud University-Computer and Information Sciences, pp. $1-22,2018$.

[5] J. E. Fowler and J. T. Rucker, "Three-dimensional wavelet-based compression of hyperspectral imagery," Hyperspectral Data Exploitation: Theory and Applications, pp. 379-407, 2007.

[6] X. Tang, et al., "Hyperspectral image compression using three-dimensional wavelet coding," SPIE/IS\&T Electronic Imaging, Proceedings of SPIE, vol. 5022, pp. 1037-1048, 2003.

[7] N. R. Kidwai, et al., "ZM-SPECK: A fast and memoryless image coder for multimedia sensor networks," IEEE Sensors Journal, vol. 16, pp. 2575-2587, 2016.

[8] H. V. Singh and A. Rai, "Medical Image Watermarking in Transform Domain," Smart Innovations in Communication and Computational Sciences. Springer, Singapore, pp. 485-493, 2019.

[9] X. Tang, et al., "Comparison of 3D set partitioning methods in hyperspectral image compression featuring an improved 3D-SPIHT," Data Compression Conference, pp. 1-4, 2003.

[10] R. Ngadiran, et al., "Efficient Implementation of 3D Listless SPECK," International Conference on Computer and Communication Engineering, Kuala Lumpur, Malaysia, pp. 1-4, 2010.

[11] S. Bajpai, et al., "3D Wavelet Block Tree Coding for Hyperspectral Images," International Conference on Engineering, Technology and Management for Sustainable Development (ICETMSD 2018), Dehradun, India, pp. $1-5,2018$.

[12] S. Jia and Y. Qian, "Spectral and spatial complexity-based hyperspectral unmixing," IEEE Trans. Geosci. Remote Sens., vol. 45, pp. 3867-3879, 2007.

[13] E. Christophe, et al., "Hyperspectral image compression: adapting SPIHT and EZW to anisotropic 3-D wavelet coding," IEEE Transactions on Image processing, vol. 17, pp. 2334-2346, 2008.

[14] A. Datta, et al., "Supervised Feature Extraction of Hyperspectral Images Using Partitioned Maximum Margin Criterion," IEEE Geoscience and Remote Sensing Letters, vol. 14, pp. 82-86, Jan 2017.

[15] V. Sowmya, et al., "Hyperspectral Image: Fundamentals and Advances," Recent Advances in Computer Vision. Springer, Cham, pp. 401-424, 2019.

[16] Z. Huilai, "A Complete Lattice Lossless Compression Storage Model," TELKOMNIKA Indonesian Journal of Electrical Engineering, vol. 12, pp. 6332-6337, 2014.

[17] P. Charan, et al., "A Cooperative Cache Management Scheme for IEEE802.15.4 based Wireless Sensor Networks," A Cooperative Cache Management Scheme for IEEE802.15.4 based Wireless Sensor Networks, vol. 8, pp. 17011710, 2018.

[18] R. K. Kumar, et al., "Dimensionality Reduction and Classification of Hyperspectral Images using Genetic Algorithm," Indonesian Journal of Electrical Engineering and Computer Science, vol. 3, pp. 503-511, 2016.

\section{BIOGRAPHIES OF AUTHORS}

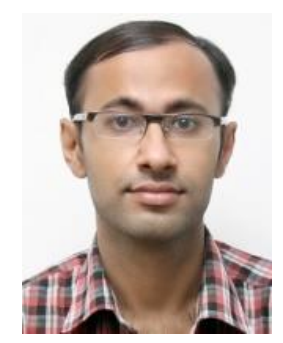

Shrish Bajpai is the doctoral student at Electronic Engineering Department, A.K.T.U., Lucknow, India. He completed his M. Tech in Digital Communication in 2012 from Bundelkhand Institute of Engineering and Technology, Jhansi, India. His research interests are hyperspectral image compression, hyperspectral feature extraction, remote sensing \& machine learning. 


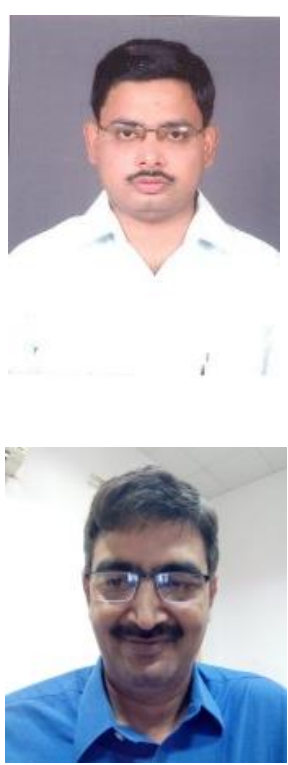

Harsh Vikarm Singh has completed his B.Tech. (Hons.) in 2002 and Ph.D. in 2008 form Indian Institute of Technology, BHU, Varanasi (U.P.) and presently he is working as Associate Professor in Kamla Nehru Institute of Technology (An Autonomous Govt. Institution), Sultanpur (U.P.), India. He is having more than 10 years of teaching and research experience. He is the principal investigator of 3 projects from Department of Science \& Technology (DST) and All India Council for Technical Education (AICTE), India of Rs. 20 Lakh. He is author of 1 book and having more than 42 international and more than 60 national publications. He has guided 45 M.Tech. students and supervised 3 Ph.D. thesis. Currently 4 Ph.D. Scholars working in his supervision. His areas of interest mainly include Digital Image Processing, Digital Watermarking, Machine Learning, Steganography, Artificial Intelligence, Cryptography Data Hiding \& Biometrics.

Naimur Rahman Kidwai received the B.Sc.(Engg.) degree in electronics engineering from the Zakir Hussain College of Engineering and Technology, Aligarh Muslim University, India, in 1996, the M.Tech. degree in digital communication from Uttar Pradesh Technical University, Lucknow, India, in 2006, and the Ph.D. degree from Integral University, Lucknow, in 2014. He is Professor at ECE Department, Integral University, Lucknow. 